\title{
Correction to: Identification of novel mutations in FFPE lung adenocarcinomas using DEPArray sorting technology and next-generation sequencing
}

Ji Won Lee ${ }^{1,2,3}$. Jong-Yeon Shin ${ }^{2,4}$ J Jeong-Sun Seo ${ }^{1,2,3,4}$

Published online: 24 April 2018

(C) Institute of Plant Genetics, Polish Academy of Sciences, Poznan 2018

Correction to: Journal of Applied Genetics 2018 https://doi.org/10.1007/s13353-018-0439-4

In the original article, part of Table 1 headings and entries were missing. The correct Table is as shown below. The original article has been corrected.

The online version of the original article can be found at https://oi.org/ 10.1007/s13353-018-0439-4

$\triangle$ Jeong-Sun Seo

jeongsun@snu.ac.kr

1 Gongwu Genomic Medicine Institute (G2MI), Medical Research Center, Seoul National University Bundang Hospital, Seongnamsi 13605, Republic of Korea

2 Genomic Medicine Institute (GMI), Medical Research Center, Seoul National University, Seoul 03080, Republic of Korea

3 Department of Biomedical Sciences, Seoul National University College of Medicine, Seoul, Republic of Korea

4 Macrogen Genome Institute, Medical Research Center, Seoul National University Bundang Hospital, Seongnamsi 13605, Republic of Korea 


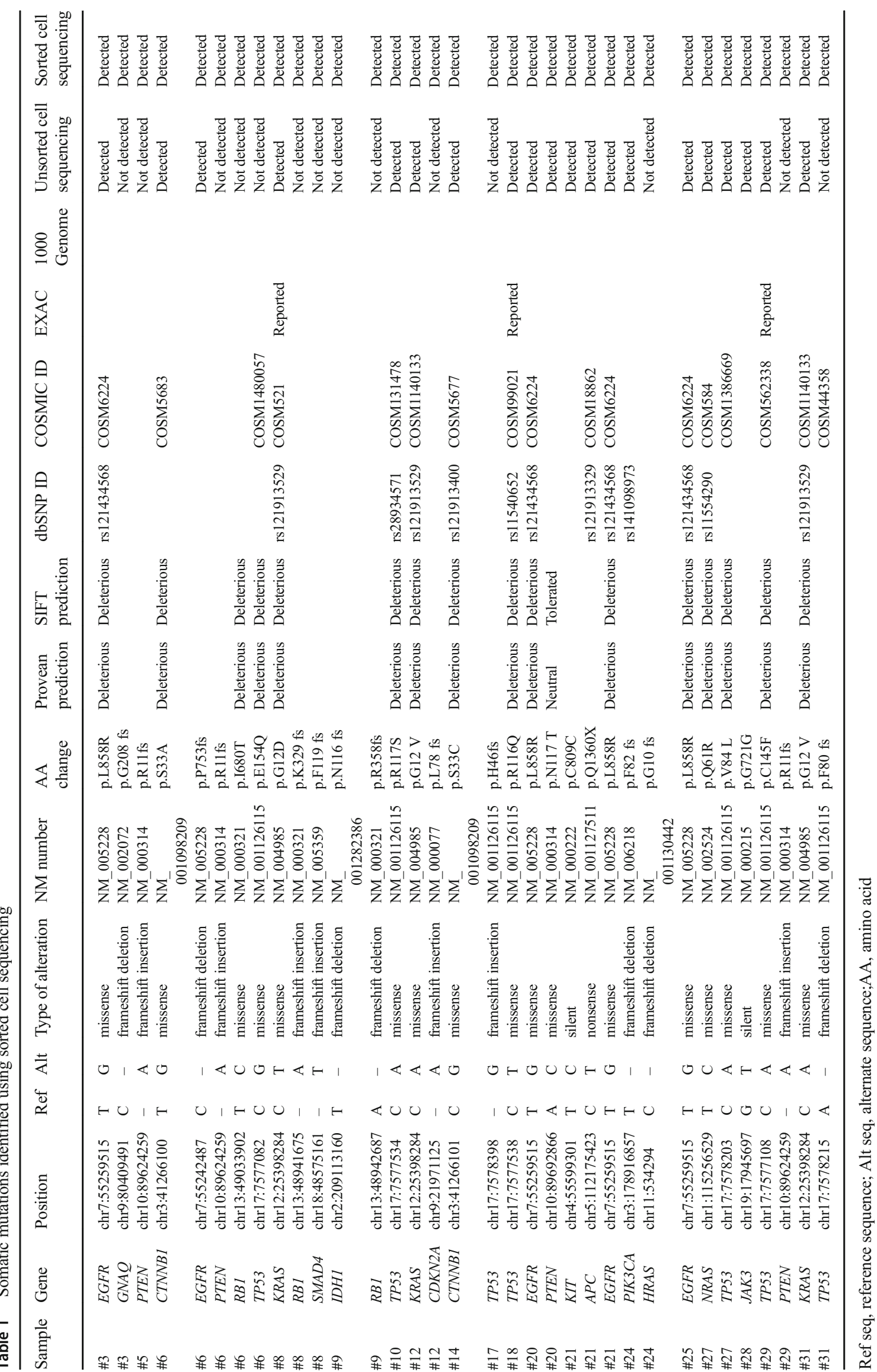

